\title{
Detecting the Unstable Points in Deformation Monitoring Geodetic Networks in Analysis Method of Subnetwork
}

\author{
Peyman Javadi ${ }^{1}$ \\ ${ }^{1}$ Master of Geodesy, Young Researcher and Elite Club, Ardabil Branch, Islamic Azad University, Ardabil, Iran \\ Correspondence: Master of Geodesy, Young Researcher and Elite Club, Ardabil Branch, Islamic Azad University, \\ Ardabil, Iran. E-mail: peyman_javadi@ardabil-samacollege.ir
}

Received: September 5, 2016

Accepted: September 26, 2016

Online Published: December 27, 2016

doi:10.5539/mas.v11n3p61

URL: http://dx.doi.org/10.5539/mas.v11n3p61

The research is financed by (Sponsoring information).

\begin{abstract}
One of the most crucial issues in engineering of structure and investigating ground deformation is deformation monitoring. The only thing which is strongly required is to create microgeodesy networks. An essential issue in microgeodesy networks is detecting unstable points of network. L1-Norm minimization and the global congruency can be noted as one of the classical methods for identifying network unstable points. In all previously conducted studies regarding this issue, results distinctly demonstrates that when displacement point vector is small, the number of points which have really displaced is more than that of true detection of displaced points using common deformation analysis ways. The probable reason for that can refer to spreading nature of the least squares estimation. Considering the results of recent studies in the detecting the network unstable points, to tackle the limitation the idea of subnetwork analysis is offered. In this case, some subnetworks including a subject point and the other source points appeared from dividing the deformation monitoring network. According to the unstable points, subnetworks will be there. This method will enable us to investigate the stable and unstable points. Having divided whole network to subnetworks, each network would be adjusted and unstable points of it would be detected. So, unstable points and their relations are cutoff and spreading effect of the least squares is fallen. This paper is on effort to evaluate the method in a simulated and a real network. The results prove that in a better and correct detection of unstable point can be successfully achieved by using subnetwork analysis compared to global congruency test all stimulates states proved the $35 \%$ of improvement on average. One percent of improvement in the results of subnetwork method to L1-Norm minimization cannot be acceptable. The algorithms of detecting unstable points in common methods and the method of analyzing subnetwork were conducted on a real network and the results are in line with simulated network results.
\end{abstract}

Keywords: deformation monitoring, congruency tests, L1- norm, subnetwork analysis, microgeodesy

\section{Introduction}

Nowadays behavior evaluation of big and sensitive structures such as dams, power plants and towers is of very high importance. Behavior survey of these structures is usually done in two geotechnical and geodetic ways. In geotechnical procedure, gauge tools of tension, shear and deflection inside the structure are installed during configuration and the data resulted from these gauges are continuously studied during and after optimization the structure in order to stability control. These tools provide the possibility of internal control of structure. In geodetic procedure, a network of points is created on the body and around the environment of structure and is monitored and controlled through geodetic observations mostly the length, angle and coordinates in different epochs. These observations provide the possibility of the deformation monitoring of the outer structure.

One of the most important geodetic network applications is deformation monitoring network or microgeodesy network. One of the important issues in these networks is the detection of stable and unstable points of network. First, by detection of stable points it can be reached to unit definition of coordinate system in two times epoch. Secondly, the deformation amount of unstable points can be calculated. If stable points are not properly identified, the calculated deformations will not valid for unstable points. In other words, the calculated deformations for network points due to the deformation of network coordinate system and isn't due to real deformation of these points. As a result, the calculated deformations can't be trusted. That is why the need for 
using appropriate productive, effective producer is felt to the detection of stable and unstable points.

In general, to consider the deformation of a network points, the awareness of points situated in two times periods and comparison of them is needed. To estimate the coordinates of the network points in each EPOCH, the least squares procedure is used. The Conventional Deformation Analysis (CDA) compares the difference of network points coordinates between two different epoch using statistical tests. If the coordinate difference is statistically significant, then the unstable point is detected. Besides, the Least Squares Estimation (LSE) is an optimal estimator, provided that the observations follow a normal distribution function. In other words, there was no wrong data in data set because LSE spread the effect of wrong data on the rest of the observations. Unfortunately, the unstable point in microgeodesy discussion also acts like error data. Therefore, the efficacy of the unstable point deformation on other spread stable points and estimated deformation will not be according to reality. In other words, when the point displaced in adjustment with the least squares procedure, the deformation effect of that point is not the only effects on that point but also effects on other points that is due to the spreading property in LSE (Chen, Kavouras \& Chrzanowski, 1987), Kuang, 1996; Prószyñski, 2000; Schwarz \& Kok, 1993).

Such as Conventional Deformation Analysis (CDA) it can be referred to Test method of global congruency test and Iterative Weighted Similarity Transformation (IWST) to find the stable points in networks. The iterative weighted similarity transformation (IWST) is done in two ways. A) Minimization L1- norm of deformation vector (LAS-L1). Minimization the second norm of deformation vector (LAS-L2). The L1- norm minimization is a powerful mathematical tool to identify the errors in mapping data. Calculation of minimization method had acceptable results. Sensitivity the first norm to the second one is in more deformation and deformations are clearer in this norm. The property of this procedure is in which it shows less sensitivity against apparent errors than the typical procedure of the least squares. In the L1- norm minimization method the efficacy of points is weighted in network datum and this weight will be appropriate to coordinate difference inverse of each point. These operations will continue because of being iterative as much the datum will be stable. It can be found by statistical test that which points have been moved at epoch time intervals. In another type of classic method i.e. the whole network stability test, first adjustment of the observations of two networks epoch is done separately. Then, it is determined by removing the efficacy of all points in datum of two networks epoch and doing statistical test that which points in datum is caused more stability. This result is obtained by testing of the whole network stability. Comparing two ways of whole network stability and minimization the first norm that was conducted by Jazayeri (1378) showed that minimization the L1- norm of the deformation vector, has more power in detection deformed points regarding to the whole network stability.

Such as new performed surveys in the field of microgeodesy, it can be referred to the performed studies in (Erdogan \& Hekimoglu, 2014; (Hekimoglu, Demirel \& Aydin, 2002; Hekimoglu, Erdogan \& Butterworth, 2010). The results of performed simulations in these studies indicate that the detected points by Convention Deformation Analysis (CDA) procedure methods are not always properly diagnosed. Due to the nature of errors spreading at the least squares the results of this study also have shown that if the network is only included one unstable point, good results will be achieved and the efficacy of errors spreading in the least squares estimation will be minimized.

According to the obtained results, it is proposed to increase the efficiency of the classic methods in microgeodesy including global congruency test method and the L1- norm minimization dividing the network to several subnetworks is used that each subnetwork includes one subject point and other reference points. Then, each of subnetworks is analyzed separately. In the present study, the data has also been implemented on simulated data on a real network.

This article contains five main parts and section. The first part discusses the introduction. The second section offer all common ways including overall network stability tests in (2-1) and the L1- norm minimization method in (2-2). The following part would be the one offered by the author himself, called subnetwork analysis method. Numerical results will be presented in section four. Section (4-1) will provide obtain results from simulated data. Section (4-2) is allocated to discuss results obtained from the real deformation monitoring network. The last section is a place to state comprehensive results and discussion

\section{Conventional Deformation Analysis (CDA) Methods}

In this section testing procedures of the global congruency test and L1-norm minimization method will be explained. Proceeding is in a way that adjusting the network in internal constraints method in two epoch observations is done separately. In this case assume that for first and the second epoch respectively we have $\left(x_{1}^{\wedge}, C_{x_{1}^{\wedge}}, d f_{1}, \sigma_{o_{1}}^{2}\right)$ and $\left(X_{2}^{\wedge}, C_{x_{2}}, d f_{2}, \sigma_{o^{2}}^{2}\right)$ where $x_{i}^{\wedge}$ is adjusted coordinates, $C_{x_{1}^{\wedge}}$ is variance covariance 
matrices of adjusted coordinates, $d f_{1}$ is the freedom degree, $\sigma_{o_{1}}^{2}$ is the estimated secondary variance factor and $i$ is the considered epoch (first or second). In order to consider the survey consistency of two epoch observations, the amount of the secondary variance factor in two epoch should be tested. Therefore the following test estimation will be used (Cooper, 1987; Chen, Chrzanowski \& Secord, 1990; Caspary, 1987):

$$
T=\frac{\widehat{\sigma}_{01}^{2}}{\widehat{\sigma}_{02}^{2}} \sim F_{d f_{1}, d f_{2}}
$$

Where in the meaningful level $\alpha$, zero hypothesis will be accepted if $T<F_{\alpha, d_{f 1}, d_{f 2}}$. Otherwise, zero hypothesis is rejected and represent the conflict between two epoch observations that often are the reason for this misinterpretation of variance factor or observations weight in adjustment. After doing the above test, in the adjusted coordinates in first epoch $X_{1}^{\wedge}$ with covariance matric $C_{X_{1}^{\wedge}}$ and second epoch $X_{2}^{\wedge}$ with covariance matrice $C_{X_{2}^{\wedge}}$, the external deformation vector is defined as:

$$
\hat{d}=\hat{x}_{2}-\hat{x}_{1}
$$

And covariance matric with correlation assumption $X_{2}^{\wedge}, X_{1}^{\wedge}$ is abtained from following equation:

$$
C_{\widehat{d}}=C_{\hat{x}_{1}}+C_{\hat{x}_{2}}
$$

The next phase is the testing of apparent deformation in network. So, the following statistical test is used.

$$
\left\{\begin{array}{l}
H_{0}: E(\hat{d})=0 \\
H_{1}: E(\hat{d}) \neq 0
\end{array}\right.
$$

The test estimation use to confirm the meaningful deformation is as follows:

$$
\omega=\frac{\hat{d}^{T} C_{d}^{+} \hat{d}}{h \widehat{\sigma}_{0}^{2}} \sim F_{h, d f}
$$

Where in this equation $\mathrm{h}=\operatorname{rank}\left(C_{d^{\wedge}}\right)$,

$\sigma_{0}^{2}=\frac{d f_{1} \sigma_{O_{1}}^{2}+d f_{2} \sigma_{O_{2}}^{2}}{d f}$ is the collective variance factor, $d f=d f_{1}+d f_{2}$ is the whole freedom degree and $f_{h, d f}$ is fisher distribution. Besides, the sign of "+" indicates Moore-Penrose invers of covariance-variance matric. The statistical test (5) indicates the stability of network (Pelzer, 1971). If $\omega>F_{h, d f, \alpha}$, then $\mathrm{E}\left(d^{\wedge}\right)=0$ i.e. the deformation is not meaningful, but if $\mathrm{w}>f_{h, d f, a}$, then $\mathrm{E}\left(d^{\wedge}\right) \neq 0$, so the zero hypothesis is rejected and the deformation is significant. In other words, the unstable datum will be detected. If in a network the deformation is significant using one of the methods of global congruency test and L1-norm minimization, first, the reference points will be detected and then the deformation values are calculated

\subsection{Overall Network Stability Tests}

This method is from the oldest methods of stable point's detection in deformation network that now is also used. For example, one of cases that this method has been used in order to the detection of points stability has been provided in reference (Hekimoglu, Demirel \& Aydin, 2002) that in this paper, it has been used to analyze the deformation of vertical network after adjusting this method.

If datum detected unstable (test 5 rejected), according to share of all points in datum, it is cleared that at least one point of this network has displacement in an internal of two epochs. First, internal constraints datum is placed between all points on network. Now it is required to find a point that has the most deformation and delete its role from datum. Therefore the datum with internal constraints between the remaining points (except deleted point or points) is selected. Now the share of different points on estimation $\omega$ should be found. To identify the point that causes network instability, the deformation share of each point such as i in formation (5), is calculated using the following equation:

$$
\Omega_{i}=\hat{d}_{i}^{T} C_{\hat{d}_{i}}^{-1} \hat{d}_{i}
$$

Where $C_{d_{1}}$ variance-covariance matric of $i^{\text {th }}$ point and deformation vector of each point is attained by following equation: 




Assuming the point $1 \leq j \leq m>j$ that $m$ is the number of network points has the highest amount for $\Omega$, this point may be one of the deformed network points. The most important point is that necessarily the biggest $\Omega_{j}$ is not belonging to the biggest $d_{j}^{\wedge}$ or the biggest $d_{j}^{T} C_{d_{j}}^{-1} d_{j}^{\wedge}$. The effect of this suspected point that has the biggest $\Omega_{j}$ should be removed from datum and $\omega$ retested. In the event of rejection again, $\omega$ on all remaining points of above steps is repeated and the point is removed from datum again. This process continues unit $\omega$ under the relationship test is not rejected. To consider other points that may have been displaced, first, it is required the point of $\mathrm{j}$ is removed from datum definition. To achieve this goal, instead of the columns related to this point in internal constraint datum matric, zero is place. To avoid the repetition of adjustment calculations, similarity transformation can be used (Cooper, 1987). A more detailed description is available in references (Baarda, 1981; Teunissen, 1985). Therefore, the deformation vector in new system and transformed variance-covariance matric respectively, are calculated by the following relations.

$$
\begin{gathered}
\hat{d}_{s}=S \hat{d} \\
C_{\hat{d}_{s}}=S C_{\widehat{d}} S^{T}
\end{gathered}
$$

That in this equation the similarity transformation is $S=I-H^{T}\left(D H^{T}\right)^{-1} D$.

In this equation, matric $\mathrm{H}$ is internal constraint datum. In relation (8), $d_{s}^{\wedge}$ is closer to real network deformation. Now, the point that has the highest value in $d_{s}^{\wedge}$ (or the same point $\mathrm{j}$ ) and has variance-covariance in matric is removed and returned again to the second step i.e. $\omega$ test and the test are as follows:

$$
\omega=\frac{d_{S}^{T} C_{d_{S}}^{+} \hat{d}_{s}}{h \widehat{\sigma}_{0}^{2}} \approx F_{h-2, d f}
$$

where $h=\operatorname{rank}\left(C_{d_{s}^{\wedge}}\right)$. If $F_{h-2, d f}$, there will be a significant deformation in network again. Therefore, the explained cases are repeated again unit $\omega$ test estimation is not rejected. The global congruency test method is explained completely by (Van Mierlo, 1978) and (Niemeier, 1981). Also, this method is performed by (Erdogan \& Hekimoglu, 2014). An interested reader can refer to mentioned references or (Cooper, 1987).

\subsection{The L1- norm Minimization Method}

In this method, the coordinates system is selected that the length of deformation vector is minimized that is called stable coordinates system (Chen, 1983). The base of this method is based on the L1-norm minimization of deformation vector. In other words, in this method, between available datum, a datum is selected in which the first of deformation vector is minimized. In mathematical way:

$$
|| d||=\sum_{i=1}^{n}|d(i)| \rightarrow \min
$$

Therefore, after the determination the vector $\mathrm{d}$, this vector is transformed to different coordinates system to clear in which system the L1- norm deformation vector has the least value passible. Again, to avoid of adjusting after the changing of coordinates system, the similarity transformation of $\mathrm{S}$ is used as $d_{s}^{\wedge}=s d^{\wedge}$ (relation 8) in which Datum matric $\mathrm{D}$ is determined as follows:

$$
D=H W
$$

Where $\omega$ is the weight matric for network coordinates system. As a result, by the placement of equation 12 in relation of similarity transformation $\mathrm{S}$ we will have: 


$$
S=I-H^{T}\left(H W H^{T}\right)^{-1} H W
$$

By repeating this procedure, the effect of unstable points in coordinates system is reduced. At first stage, weight matric is matric I named $w^{1}=\mathrm{I}$, where deformation vector will be $d_{s}^{\wedge}=S^{I} \alpha^{\wedge}$. Considering that it is necessary that the weight of datum points with displacement has inverse proportion, matric $\omega$ is a diagonal matric that components $i^{\text {th }}$ of main diameter is $\frac{1}{d_{s}^{\wedge}(i)}$. Due to it is possible that deformation components is some places or at least one of zero coordinates components is obtained, the denominator will be zero, therefore, it is necessary to avoid of zero denominator. In fact, the weight matric structure in the next irritations changes as follows:

$$
W_{j j}^{(i)}=\operatorname{diag}\left(\frac{1}{\left|\hat{d}_{s}^{(i-1)}(j)\right|+\varepsilon}\right), j=1, \ldots, n
$$

Where $\varepsilon$ is a small positive number that avoid od zero denominator. By this definition of $\mathrm{w}$, in fact, the points that has small deformation will acquire a larger share in Datum an reverse the points that are with larger deformation, has will the smaller share in Datum. i.e.

$$
\begin{gathered}
W^{(1)}=I \\
S^{(i)}=I-H^{T}\left(H W^{(i)} H^{T}\right)^{-1} H W^{(i)} \\
\hat{d}_{s}=S^{(i)} \hat{d} \\
W_{j j}^{(i)}=\operatorname{diag}\left(\frac{1}{\left|\hat{d}_{s}^{(i-1)}(j)\right|+\varepsilon}\right), j=1, \ldots, n \\
\text { repeat until }\left|\hat{d}_{s}^{(i)}-\hat{d}_{s}^{(i-1)}\right|<\delta
\end{gathered}
$$

The important point in the provided process in 15 relations is the similarity transformation $\mathrm{S}$ on each of $d_{s}^{\wedge}$ or first $d^{\wedge}$ applied, it has no difference, because $S_{i} S_{k} S_{j}=S_{i}$, named the similarity transformation $\mathrm{S}$ is the transformation of that exponent. The repetition continues until the vector difference $\hat{d}$ is reduced in two successive stages than a desired specified limit, i.e.

$\left|d_{s}^{\wedge(i)}-d_{s}^{\wedge(i-1)}\right|$ that usually a small number is selected for $\delta$ i.e 0.5 or $0.1 \mathrm{~mm}$. finally after determining the weight points in network datum named completing the relations loop (15) that selected the final datum in a way that the L1-norm of the points deformation vector is minimized, the detection test of stable an unstable points as follows:

$$
F_{i}=\frac{d_{s_{i}}^{T} c_{d_{i}}{ }^{-1} d_{s_{i}}}{C \widehat{\sigma}_{0}^{2}} \approx F_{c, d f}, i=1, \ldots k
$$

Where $\mathrm{k}$ is the point number and $d_{s i}$ is coordinates components of deformation vector related to each point after transformation by helping of the similarity transformation $\mathrm{S}$ each $\mathrm{C}$ is equal to network to network dimension. This test is done on the individual points to determine each point is stable or not? If $\mathrm{f}_{1}>\mathrm{f}_{\mathrm{c}, \mathrm{df}, \mathrm{a}}$ the point $i^{\text {th }}$ is displaced (is removed as unstable point), otherwise the point is stable. More details about this method is available in (Chen, 1983; Chen, Chrzanowski \& Secord, 1990; Setan, 1995; Setan, \& Sing, 2001; Taşçi, 2010) resources.

\subsection{Subnetwork Analysis Method}

The conventional deformation analysis (CDA), the coordinates statistical test is compared. Id coordinates difference shows deformation title. In the previous sections, two examples of CDA common methods were explained. The results of performed simulations in recent researches suggests that detected points by CDA is not always identified correctly (Hekimoglu, Erdogan \& Butterworth, 2010). That is the reason can be the same as spreading property of LSE. Because the importance of this section and using of proposed idea in this research, the summary of research (Erdogan \& Hekimoglu, 2014) and (Hekimoglu, Erdogan \& Butterworth, 2010) is presented. 
In paper (Hekimoglu, Erdogan \& Butterworth, 2010), the obtained results for performed simulations (simulations in 1000 times) shown when applied deformation is small, the number of correct points detection using of CDA methods is less than the number of points that were really displaced. The reason of it can be LSE spreading property (Hekimoglu, Erdogan \& Butterworth, 2010). Therefore, the optional applied deformation degree between $r$ and $2 r$ and again between $r$ and $3 r$ is selected. (r) is a number that by changing it, the success rate of correct detection from unstable points is 81.3 percent that is a value for efficiency measurement of CDA methods when a point is displaced and optional applied deformation is between rand 2r. These results are not satisfactory, the results of this study also indicated that if the network only has one stable point, good results is achieved and the efficacy of LSE spreading is minimized. Therefore, according to this point, the idea that has been presented in this paper is the division of overall network to multi-subnetwork so that each unstable point is placed on individual subnet and then is checked. In fact, every subnet includes one subject point and other referenced points. In this method, the relation between unstable points is interrupted from each other and finally effect of spreading the least squares is removed. Through this method, if there are 3 subject points (A, B, C) in a network, so we will have subnet i numbers of subject points. Subnetwork I is subnet that only includes one and other reference points (not include B, C points). At the same way, the second subnetwork includes B point and other reference points and the third subnetwork includes $\mathrm{C}$ point and other points. Each subnet is analyzed separately. What is clear from the obtained results is that in network division to subnet, better results have been obtained. Therefor subnet method is advised rather than the global congruency. In this stable to increase the efficiency the present classic method in micro geodesy such as the global congruency test, network division to multi subnet has been used. Then each is adjusted separately in internal constraints method and classic method of the overall stability test to detect stable and unstable points of network has been used. These calculations in each epoch, once for overall network and another time for three subnetworks are done and each of them has been compared to their first epoch. What is presented from the results and better results has been obtained from network division to subnet. According to obtained results is this paper, the efficiency survey of present classic method in microgeodesy such as overall stability test method and L1-norm minimization in two ways of overall analysis and subnet analysis using simulated date and real network observation will be discussed.

3. Results

\subsection{Numerical Results}

In this section, the global congruency test methods and the L1- norm minimization in two ways of subnet analysis and the whole network analysis will be compared. Therefore, simulation observations of GPS three dimensional network have been used. Moreover, the possible displacement will be determined by using real network observations and GPS observations.

\subsection{Simulated Data}

In order to evaluate the performance of proposed method from subnet analysis compared to current method of overall network analysis, simulated data can be used in which first, deformation points, rates and deformation direction are applied optionally then the rate and percentage of correct detection of unstable points will be determined by using the algorithms of unstable points detection. According to common usage of GPS observations is microgeodesy, the applied simulated observation in this study, is GPS length-based observation. The network is a three-dimensional network including 8 points (5points as reference or stable points and 3 subject points). All present base lines are considered between points as the observations that total number of base lines is 28 (equal to 84 observations) in discussed 8 point network. To ensure the results, the observations are simulated 1000 times. In order to create simulated observations in two epochs, first the observations without error are calculated, and then the error rate with normal distribution to zero mean and standard deviation (SEM) $5 \mathrm{~mm}$ is added. Therefore, these observations are the first epoch observations. After making the first the first epoch observations, it is required to change the point and make simulated observations for second epoch according to explained method. It is considered different ways to simulate that related results to each simulated ways in sections 3.2.1, 3.2.2, 3.2.3, 3.2.4 will be presented respectively.

\subsubsection{Regular Network with Definite Deformations}

In the first state, a regular network including 8 points ( 5 points as reference or stable points and 3 subject points) was presented. Reference points of this network (points 1-5) are placed on a circle with a radius 200 and in the center $(500,500)$. The displacements of deformed points were supposed zero and the obtained were examined. Table 1 indicates the coordinates of network simulated points. Figure (1-A) indicates a view of simulated network. In this figure, the points 1-5 are reference or stable points and points OBJ1, OBJ2, OBJ3 are subject points. 
Table 1. Coordinates of network simulated points

\begin{tabular}{cccc}
\hline $\mathrm{Z}(\mathrm{m})$ & $\mathrm{Y}(\mathrm{m})$ & $\mathrm{X}(\mathrm{m})$ & point \\
\hline 500 & 500 & 700 & 1 \\
\hline 510 & 690.211 & 561.803 & 2 \\
\hline 495 & 617.557 & 338.197 & 3 \\
\hline 490 & 382.443 & 338.197 & 4 \\
\hline 515 & 309.789 & 561.803 & 5 \\
\hline 520 & 540 & 510 & OBJ1 \\
\hline 505 & 430 & 600 & OBJ2 \\
\hline 525 & 480 & 380 & OBJ3 \\
\hline
\end{tabular}

According to the presented idea and to reduce the effect of error distribution (spreading) in the least squares, this overall network is divided to three subnetworks. Each of them only contains one of the subject points. Figure (1) indicates the first, second and third subnetworks. The observations of GPS base line includes two epochs that in the second epoch, the points of subject points are displaced optionally and the displaced points are detected using the overall network analysis and subnetwork. In this study, three different scenarios are done and compared with the first epoch. These three scenarios are as follows:

1- OBJ1 and OBJ2 are displaced.

2- OBJ2 and OBJ3 are displaced

3- All three points of OBJ1, OBJ2 and OBJ3 are displaced at the same time.

Table 2. Indicates the displaced amounts in each scenario.

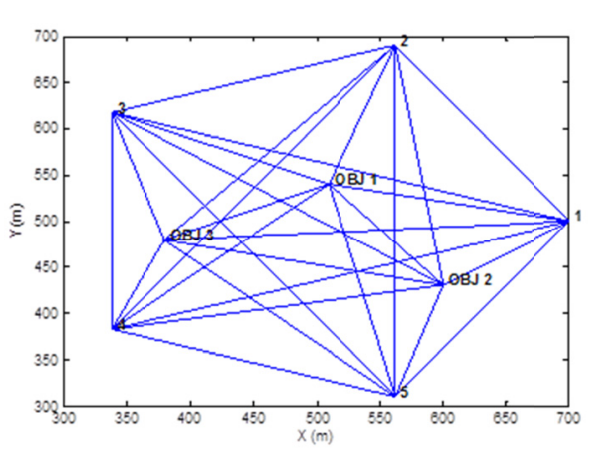

(A)

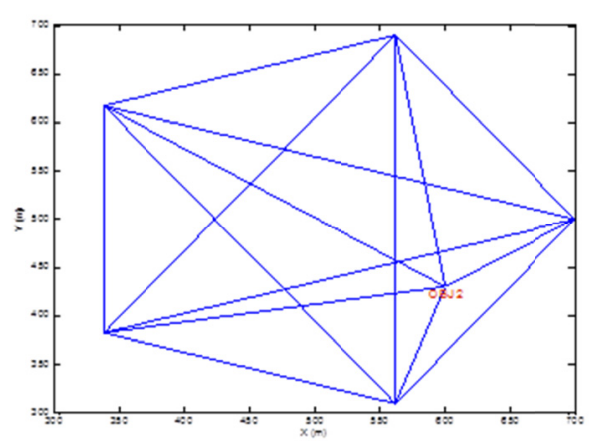

(C)

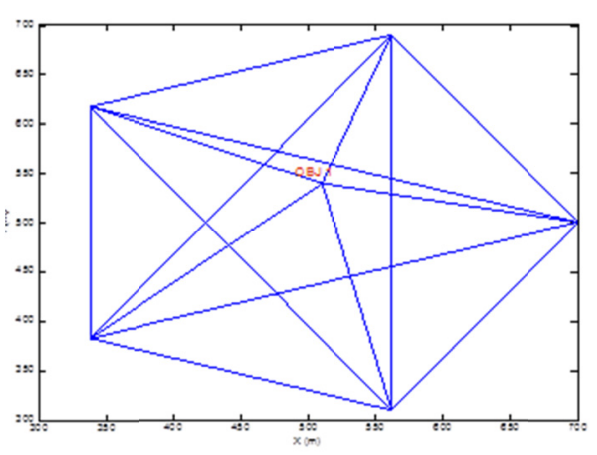

(B)

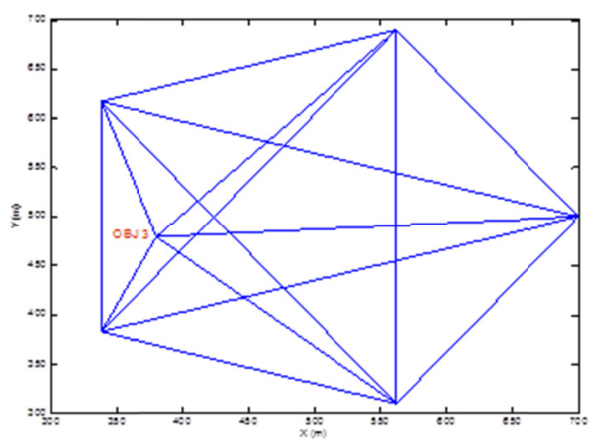

(D)

Figure 1. (A) The simulated network contains 8 points ( 5 points as reference points and 3 subject points), (B) The first subnetwork contains OBJ1 subject point and other reference points, $(C)$ the first subnetwork contains OBJ2 
subject point and other reference points, (D) the first subnetwork contains OBJ3 subject point and other reference points

Table 2. The point's deformation in meters in different scenarios

\begin{tabular}{ccccc}
\hline$\Delta \boldsymbol{Z}$ & $\Delta \boldsymbol{Y}$ & $\boldsymbol{\Delta} \boldsymbol{X}$ & Displaced points & scenario \\
\hline-0.008 & 0.008 & 0.009 & OBJ1 & \multirow{2}{*}{1} \\
\cline { 1 - 4 }-0.008 & 0.009 & 0.009 & OBJ2 & \\
\cline { 1 - 4 }-0.008 & 0.007 & 0.009 & OBJ2 & \multirow{2}{*}{2} \\
\hline-0.008 & 0.008 & 0.009 & OBJ3 & \\
\cline { 1 - 4 }-0.009 & 0.008 & 0.009 & OBJ1 & \\
\cline { 1 - 3 } 0.007 & 0.007 & 0.008 & OBJ2 & \\
\hline 0.007 & 0.006 & 0.006 & OBJ3 & \\
\hline
\end{tabular}

After the simulation of observations in two epoch in three different scenarios, individual adjusting of two epochs in order to usage of overall stability test methods and minimization of L1-norm in two ways of overall network analysis and subnetwork is done to detect displaced points. The approach is that first the network is adjusted in internal constraints method then classic method of global congruency test and L1-norm minimization have been used to detect stable and unstable points of network. These calculations in each epoch are done once for the whole network and another time for three subnetworks and each network is compared to its first epoch. Then the detected points by these two methods are compared with the points that are really displaced. In this way, the performance of these two methods will be compared with each other in unstable point's detection. As it said, in order to ensure the results, it will be repeated 1000 times. Table (3) indicates the summary of results in unstable point's detection in two global congruency test method and L1-norm minimization in two ways of overall network analysis and subnetwork in all three scenarios.

Table 3. Correct detection percentage of unstable points in regular network with definite deformations

\begin{tabular}{ccc}
\hline Detection percentage of unstable points & Detection methods of unstable points & scenario \\
\hline $34.3 \%$ & Global congruency test method & \\
$59.4 \%$ & L1-norm minimization method & First \\
$54.3 \%$ & Subnetwork method with Global congruency test method & \\
$62.2 \%$ & Llobal congruency test method & \\
$30.7 \%$ & Subnetwork method with L1-norm minimization method & \\
$59.6 \%$ & Subnetwork method with Global congruency test method & Second \\
$48.5 \%$ & Subnetwork method with L1-norm minimization method & \\
$61.2 \%$ & Global congruency test method & \\
$1.4 \%$ & L1-norm minimization method & Third \\
$34.8 \%$ & Subnetwork method with Global congruency test method & \\
$22.6 \%$ & & \\
$36.4 \%$ & &
\end{tabular}

As it is clear from table (3), in the first scenario in using of subnetworks instead of overall network, in both Global congruency test method and in the L1-norm minimization method, the improvement will be achieved. Especially in Global congruency test, the improvement is equal 20 percent and in the L1-norm minimization method the improvement equal to 3 percent will occurred. Also in second scenario in Global congruency test method the improvement will equal to 18 percent and in L1-norm minimization method it will equal to 2 percent. Finally, in the third scenario in Global congruency test method the improvement will be 22 percent and in the L1-norm minimization method it will be 2 percent

\subsubsection{Regular Network with Random Deformations}

In this part we will investigate the results on the same designed regular network of pervious section ended with random deformations between 8 to $10 \mathrm{~mm}$ (positive or negative that in each program running will be randomly selected). Here, three different scenarios are performed and compared with the first epoch. 
1- $\quad$ OBJ1 and OBJ2 points are displaced (randomly).

2- $\quad$ OBJ2 and OBJ3 are displaced (randomly).

3- $\quad$ All three points of OBJ1, OBJ2 and OBJ3 are displaced simultaneously (randomly).

Table 4. Indicates the summary results in unstable point's detection in two methods of global congruency test method and L1-norm minimization method in two ways of overall network analysis and subnetwork.

Table 4. Correct detection percentage of unstable points in regular network with random deformations

\begin{tabular}{ccc}
\hline Detection percentage of unstable points & Detection methods of unstable points & scenario \\
\hline $47.4 \%$ & Global congruency test method & \\
$68.8 \%$ & L1-norm minimization method & First \\
$82.5 \%$ & Subnetwork method with Global congruency test method & \\
$68.7 \%$ & Global congruency test method & \\
$46.6 \%$ & L1-norm minimization method & Second \\
$72.1 \%$ & Subnetwork method with L1-norm minimation method & \\
$82.7 \%$ & Subnetwork method with Global congruency test method & \\
$69.1 \%$ & Global congruency test method & \\
\hline $1.4 \%$ & L1-norm minimization method & Third \\
$65.0 \%$ & Subnetwork method with Global congruency test method & \\
$76.2 \%$ & Subnetwork method with L1-norm minimization method & \\
$64.9 \%$ & & \\
\end{tabular}

As it is clear, in the first scenario in usage of subnetwork instead of overall network, global congruency test method an improvement is achieved. While in this way, in the L1-norm minimization, the improvement is not considered. This is while the results improvement in global congruency test method is about 35 percent. Also in second scenario using subnetwork instead of overall network only in global congruency test method caused an improvement in results and detection percentage. However, in global congruency test method an improvement about 36 percent will be occurred. Finally, in third scenario in using subnetwork instead of global congruency test method an improvement about 74 percent will be occurred while an apparent difference in the L1-norm minimization is not considered.

\subsubsection{Irregular network with definite displacements}

In this section, irregular network including 8 points (5 points as reference or stable points and 3 subject points) is designed. The deformations of displaced points were assumed stable and the obtained results were examined. In this section also three different scenarios are performed according to the amounts. Table 5 indicates the results summary in unstable point's detection in two global congruency test method and L1-norm minimization in two methods of overall network analysis and subnetwork in all three scenarios.

Table (5) - correct detection percentage of unstable points in irregular network with definite displacements

\begin{tabular}{lll}
\hline $\begin{array}{l}\text { Detection } \\
\text { percentage } \\
\text { unstable } \\
\text { points }\end{array}$ & Detection methods of unstable points & scenario \\
\hline $\mathbf{3 3 . 1 \%}$ & Global congruency test method & First \\
\hline $\mathbf{6 5 . 5 \%}$ & L1-norm minimization method & \\
\hline $\mathbf{5 4 . 5 \%}$ & $\begin{array}{l}\text { Subnetwork method with Global congruency test } \\
\text { method }\end{array}$ & $\begin{array}{l}\text { Subnetwork method with L1-norm minimization } \\
\text { method }\end{array}$ \\
\hline $\mathbf{6 1 . 0 \%}$ & Global congruency test method & Second \\
\hline $\mathbf{3 3 . 8 \%}$ & &
\end{tabular}




\begin{tabular}{lll}
\hline $\mathbf{6 0 . 2 \%}$ & L1-norm minimization method \\
\hline $\mathbf{5 3 . 2 \%}$ & $\begin{array}{l}\text { Subnetwork method with Global congruency test } \\
\text { method }\end{array}$ & $\begin{array}{l}\text { Subnetwork method with L1-norm minimization } \\
\text { method }\end{array}$ \\
\hline $\mathbf{6 1 . 0 \%}$ & Global congruency test method & Third \\
\hline $\mathbf{1 . 2 \%}$ & L1-norm minimization method \\
\hline $\mathbf{3 6 . 3 \%}$ & $\begin{array}{l}\text { Subnetwork method with Global congruency test } \\
\text { method }\end{array}$ & $\begin{array}{l}\text { Subnetwork method with L1-norm minimization } \\
\text { method }\end{array}$ \\
\hline $\mathbf{3 7 \%}$ & & \\
\hline
\end{tabular}

As it is clear from table (5), in the first scenario in using subnetwork instead of overall network, the improvement will be occurred in both global congruency test method and L1-norm minimization method. However, this improvement in L1-norm minimization method is 5\% percent but in global congruency test method it is increased 21 percent. In second scenario also using subnetwork instead of overall network is caused the improvement in the results or detection percentage in both global congruency test method and L1-norm minimization method. In this section, the improvement in L1-norm minimization is $8 \%$ but in global congruency test method is increased $15 \%$. Therefore, it can be said that using subnetwork in global congruency test causes to results improvement. Finally, in third scenario also in using subnetwork instead of overall network in both global congruency test method and L1-norm minimization, the improvement will be achieved. The improvement of global congruency test method is about 22 percent and L1-norm minimization method is about 7 percent that is not so significant.

\subsubsection{Irregular network with random displacements}

In this section, an irregular network including 8 points ( 5 points as reference or stable points and 3 subject points) is designed. The deformations of random points will be placed between 8 to $10 \mathrm{~mm}$ (positive or negative). In this section also three different scenarios are performed according to 4-1-2 section. Table 6 indicates the results summary in unstable point's detection in two ways of global congruency test method and L1-norm minimization method in two methods of overall network analysis and subnetwork in all three scenarios. As it is clear from table 6 , in first scenario in using of subnetwork instead of overall network, global congruency test method an improvement about 36 percent has been achieved. In second scenario also, the use of subnetwork instead of overall network will be about 37 percent only in global congruency test method and is caused an improvement in results and detection percentage. Finally, in third scenario in using subnetwork instead of overall network in global congruency test method, an improvement about 84 percent will be occurred while in L1-norm minimization, apparent difference is not considered.

Table (6) - correct detection percentage of unstable points in irregular network with random displacements

\begin{tabular}{lll}
\hline $\begin{array}{l}\text { Detection } \\
\text { percentage of } \\
\text { unstable } \\
\text { points }\end{array}$ & Detection methods of unstable points & scenario \\
\hline $\mathbf{5 3 . 7 \%}$ & Global congruency test method & First \\
\hline $\mathbf{7 2 . 0 \%}$ & L1-norm minimization method \\
\hline $\mathbf{8 8 . 8 \%}$ & $\begin{array}{l}\text { Subnetwork method with Global congruency test } \\
\text { method }\end{array}$ \\
\hline
\end{tabular}




\begin{tabular}{|c|c|c|}
\hline $68.0 \%$ & $\begin{array}{l}\text { Subnetwork method with L1-norm minimization } \\
\text { method }\end{array}$ & \\
\hline $501.0 \%$ & Global congruency test method & Second \\
\hline $71.5 \%$ & L1-norm minimization method & \\
\hline $86.9 \%$ & $\begin{array}{l}\text { Subnetwork method with Global congruency test } \\
\text { method }\end{array}$ & \\
\hline $65.0 \%$ & $\begin{array}{l}\text { Subnetwork method with L1-norm minimization } \\
\text { method }\end{array}$ & \\
\hline $1.5 \%$ & Global congruency test method & Third \\
\hline $63.5 \%$ & L1-norm minimization method & \\
\hline $86.1 \%$ & $\begin{array}{l}\text { Subnetwork method with Global congruency test } \\
\text { method }\end{array}$ & \\
\hline $62.1 \%$ & $\begin{array}{l}\text { Subnetwork method with L1-norm minimization } \\
\text { method }\end{array}$ & \\
\hline
\end{tabular}

Finally, according to the tables $(3,4,5,6)$ the following results were obtained:

A) According to the results of other studies, the L1-norm minimization method is of better capability than Global congruency test method in overall network analysis (the current methods) in the detection of unstable points.

B) Using of subnetwork method instead of overall network is caused an improvement of results in global congruency test method especially when all three subject points are displaced, in all simulated ways (regular and irregular network with definite and random displacements), the improvement of correct percentage results of point's detection is considerable. This improvement is averagely about 35 percent in all methods. However, improvement in L1-mnorm minimization is about 1 percent that cannot be considered as a significant improvement.

\subsection{The real deformation monitoring network}

After presentation of the results related to the simulated data, now the performance of proposed method is examined using the real observations of deformation monitoring network. GPS created network around Kabudval dam is located in Golestan province. The used microgeodesy network in this study includes 6 pillars $(\mathrm{KL} 1, \mathrm{KL} 2, \mathrm{KL} 3, \mathrm{KR} 1, \mathrm{KR} 2, \mathrm{KR} 3)$ that two pillars in left side, one pillar in right side, one pillar above the dam were created. Figure (2) indicates the location of these stations. The performed observations are GPS length based. The used receivers of two frequency satellite GPS receiver type are of system 500 and 1200 Leica. The observations of the first and second epoch respectively are done in the dates of 17 to 22 December 2012 and the early March 2013, i.e. at about three months interval.

The process is that observations between network points by GPS receiver is collected and processed. First, the observations of two epochs were adjusted separately and then were tested by classic methods of unstable point's detections of global congruency test method and L1-norm minimization method. Table 7 indicates the obtained results. 


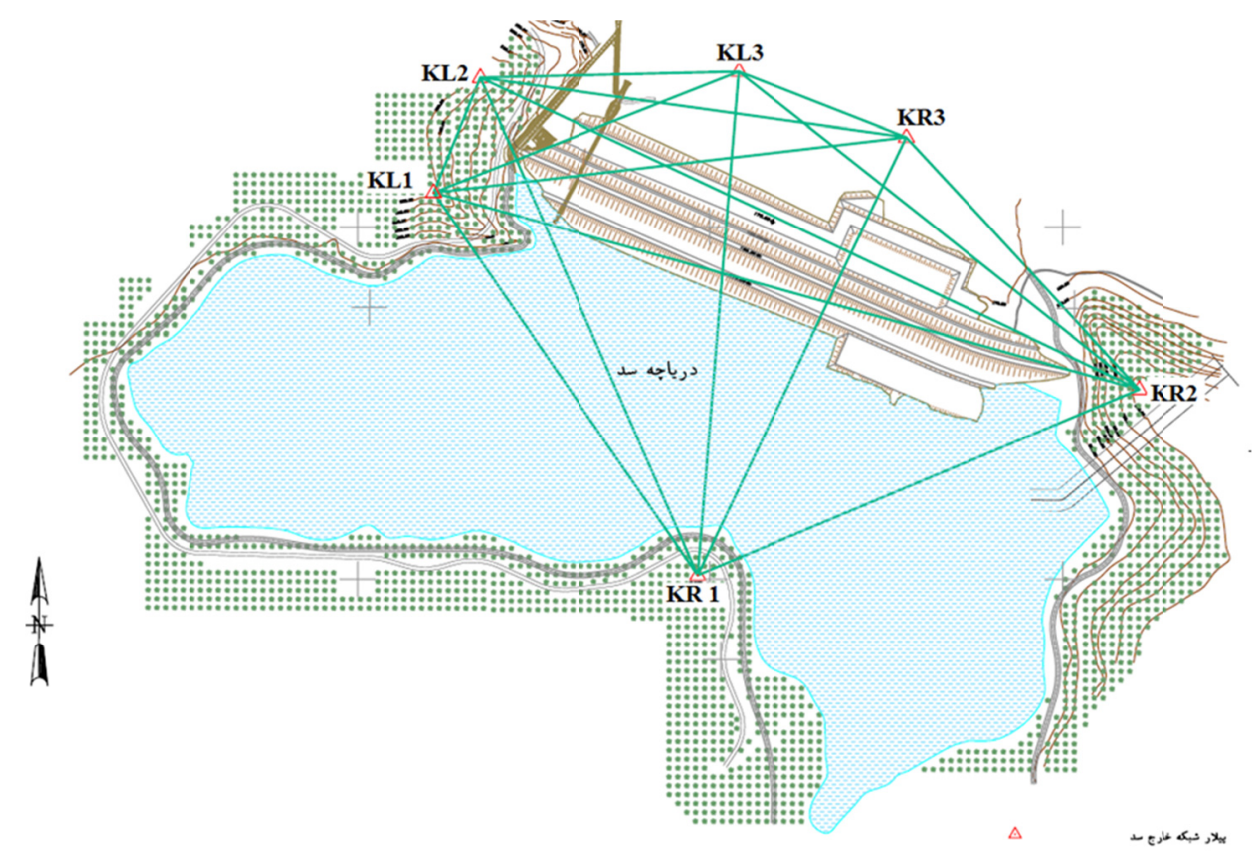

Figure 2. Microgeodesy network points which are located around dam

Table 7. The obtained results from two global congruency test and L1-norm minimization method

\begin{tabular}{ccc}
\hline \multicolumn{2}{c}{ KL1,KL2,KL3,KR1,KR2,KR3 } & All network points \\
\hline Stable Points & Detected Unstable Points & Method \\
KL2,KR1,KR2 & KL1,KL3,KR3 & global congruency test \\
KL2,KR1,KR2 & KL1,KL3,KR3 & L1-norm minimization \\
\hline
\end{tabular}

According to table (7), the results of both methods are the same and in both methods the points KL1, KL3, KR3 were detected as unstable points. Then by considering three points KL1, KL3, KR3 as unstable point's three subnetworks were analyzed and the results related to each subnetwork is presented. Therefore, the points KL1, KL2 are KR3 are selected as unstable points and three subnetworks will be created:

- The first subnetwork including KL1 point and based points KL2, KR1 AND KR2.

- The second subnetwork including KL3, point and based points KL2, KR1 and KR2.

- The third subnetwork including KR3 point and based points KL2, KR1 and KR2.

Then three subnetworks are analyzed separately and the results related to each subnetwork are presented. By using it, we want to check if really KL1, KL3 and KR3 points are stable and if the based points KL2, KR1, KR2 are really stable or not. Table (8) indicates the obtained results from subnetwork analysis $(1,2,3)$. According to table (8) it can be concluded that KR2 point is also as unstable point that not identified by overall network analysis because as it was said it can be the property of errors spreading in the least squares estimation.

Table (8) - The results obtained from first, second and third subnetworks analysis

\begin{tabular}{|c|c|c|c|}
\hline $\begin{array}{l}\text { Detected } \\
\text { points }\end{array}$ & unstable & method & subnetwork \\
\hline KL1,KL2 & & Global congruency test & \multirow{2}{*}{$\begin{array}{l}\text { First (unstable point } K L 1 \text {, and based points } \\
\mathrm{KL} 2, \mathrm{~K} R 1, \mathrm{~K} 2 \mathrm{2})\end{array}$} \\
\hline KL1,KL2 & & L1-norm minimization & \\
\hline KL3,KR2 & & Global congruency test & \multirow[t]{2}{*}{ Second (unstable point KL3, based points KL2,KR1,KR2) } \\
\hline KL3,KR2 & & L1-norm minimization & \\
\hline KR3,KR2 & & Global congruency test & Third (unstable point KR3, based points KL2,KR1,KR2) \\
\hline
\end{tabular}




\section{KR3,KR2 \\ L1-norm minimization}

Finally, unstable point's deformation will be concluded then oval of error point is drawn with deformation vector of point in 95 percent confidence level. The detected unstable points are: KL1, KL3, KR3, KR2. To calculate the deformation level of these points simultaneous two epochs is used so that 4 detected unstable points are considered as two points (one point in first epoch and another new point are considered) and simultaneous adjusting of observations is presented.

Table (9) indicates deformation level of detected unstable points in north, east and up direction. Finally oval dimension of network point's error is presented in confidence level \%95 perfect in table (10).

Table (9) - The deformation of unstable points in north, east and up direction in meters

\begin{tabular}{llll}
\hline Up(mm) & North $(\mathrm{mm})$ & East $(\mathrm{mm})$ & point \\
\hline $\mathbf{- 7 . 1 7}$ & 2.57 & -6.79 & KL1 \\
\hline $\mathbf{4 . 5 9}$ & -0.55 & 2.71 & KL3 \\
\hline $\mathbf{- 8 . 4 4}$ & -1.57 & -3.41 & KR2 \\
\hline$-\mathbf{9 . 9 3}$ & -2.52 & -0.84 & KR3
\end{tabular}

Table (10) - the error oval dimensions in security level of \%95 in $\mathrm{mm}$

\begin{tabular}{lll}
\hline $\mathbf{b}(\mathbf{m m})$ & $\mathbf{a}(\mathbf{m m})$ & Point \\
\hline $\mathbf{2 . 1 3}$ & 2.61 & KL1 \\
\hline $\mathbf{1 . 9 7}$ & 2.46 & KL3 \\
\hline $\mathbf{2 0 . 0 9}$ & 2.58 & KR2 \\
\hline $\mathbf{2 . 0 2}$ & 2.49 & KR3 \\
\hline
\end{tabular}

Finally, the oval of absolute error of points in security level of $\% 95$ percent is drawn by the unstable points of deformation vector is LG system figure (3) to clear more the oval dimension is multiplied in 50.



Figure 3. The oval the error detected unstable points in security level of 95 percent by deformation vector 


\section{Summary and Conclusion}

In this study the practical survey of efficacy of two stable point's detection (global congruency test method and L1-norm minimization of deformation vector) in deformation monitoring networks as subnetwork analysis has been evaluated. For this purpose, global congruency test and L1-norm minimization were performed in two overall network and subnetwork analysis on some simulated data and the results were compared. The simulated observations, GPS length base observations were considered. As it is clear from the results taken from simulated network, the use of subnetwork analysis method instead of global congruency test method will cause to the improvement of the results. In subnetwork analysis method in all simulated forms (regular and irregular network with definite and random deformations), the improvement of correct results of points detection is considerable percent. This improvement in all simulated forms is averagely about 35 percent. According to the improvement of subnetwork method results is about 1 percent than L1-norm minimization that cannot be accounted it significant. In following unstable points detection algorithms, the current methods and subnetwork analysis method on observations of a real network around Kabudval dam located in Golestan province were performed that the obtained results are according to simulated network results and in the end, deformation level of detected unstable points was calculated

\section{References}

Baarda, W. (1981). S-Transformations and criterion matrices. (2nd Ed.), Publications on geodesy, Delft.

Caspary, W. F. (1987). Concepts of Network and Deformation Analysis. (1st. Ed.), School of Surveying, The University of New South Wales, Kensington.

Chen, Y. Q. (1983). Analysis of deformation surveys - A generalized method. Ph.D. Thesis, University of New Brunswick, Canada.

Chen, Y. Q., Chrzanowski, A., \& Secord, J. M. (1990). A Strategy for the analysis of the stability of reference points in deformation surveys. Canadian Institute of Surveying and Mapping journal, 44(2), 141-149.

Chen, Y. Q., Kavouras, M., \& Chrzanowski, A. (1987). A strategy for detection of outlying observations in measurements of high precision. Can, Surveyor, 41(4), 529-540.

Cooper, M. A. (1987). Control survey in civil engineering. London: William Collins Sons \& Co. Ltd.

Erdogan, B., \& Hekimoglu, S. (2014). Effect of subnetwork configuration design on deformation analysis. Survey Review, 46(335), 142-148.

Fraser, C., \& Gruendig, L. (1984). The analysis of photogrammetric deformation measurements on Turtle Mountain. Photog. Engr. and Remote Sending, 51(2), 207-216.

Hekimoglu, S., Demirel, H., \& Aydin, C. (2002). Reliability of the Conventional Deformation Analysis Methods for Vertical Networks. FIG XXII International Congress, Washington, D.C., 19-26 April 2002.

Hekimoglu, S., Erdogan, B., \& Butterworth, S. (2010). Increasing the efficacy of the conventional deformation analysis methods: alternative strategy. Journal of Surveying Engineering, 136(2), 53-62.

Jazaeri, J. S. Sh. (2008). Comparing congruency robust method and L1 norm minimization in microgeodesy networks. Journal of the Earth and Space Physics, 34(4), 117-132.

Kuang, S. (1996). Geodetic network analysis and optimal design: Concepts and applications. (1st. Ed.), Ann Arbor Press, Inc, Michigan.

Niemeier, W. (1981). Statistical tests for detecting movements in repeatedly measured geodetic networks. Journal of Tectonophysics, 71(1-4), 335-351.

Pelzer, H. (1971). Zur Analyse geodätischer Deformationsmessungen. (1st, Ed.). Verlag der Bayer. Akad. d. Wiss. München.

Prószyñski, W. (2000). On outlier-hiding effects in specific Gauss-Markov models:geodetic examples. $J$. Geodesy, Berlin, 74(7-8), 581-589.

Schwarz, R. C., \& Kok, J. (1993). Blunder detection and data snooping in LS and robust adjustments. J. Surv. Eng., 119(4), 127-136.

Setan, H. (1995). Functional and stochastic models for geometrical detection of spatial deformation in engineering: a practical approach. Ph.D. Thesis, City University, London.

Setan, H., \& Sing, R. (2001). Deformation analysis of a geodetic monitoring network. Geomatica, 55(3), 333-346. 
Taşçi, L. (2010). Analysis of dam deformation measurements with the robust and non-robust ethods. Scientific Research and Essays , 5(14), 1770-1779.

Teunissen, P. J. G. (1985). Zero order design: Generalized inverses, adjustments, the datum problem and S-transformations. In: Grafarend E. W., Sanso F (eds), Optimization and design of geodetic networks. Springer, Berlin Heidelberg New York, 11-55.

Van Mierlo, J. (1978). A testing procedure for analysing geodetic deformation measurements. In: 2nd F.I.G. Symposium: Deformation Measurements by Geodetic Methods, Bonn.

\section{Copyrights}

Copyright for this article is retained by the author(s), with first publication rights granted to the journal.

This is an open-access article distributed under the terms and conditions of the Creative Commons Attribution license (http://creativecommons.org/licenses/by/4.0/). 